\title{
Dificultades en el desarrollo de la Ley 100
}

\author{
La respuesta de los médicos colombianos en general y de los ginecólogos en particular frente al \\ impacto que el nuevo sistema de salud ha tenido en el ejercicio profesional, no será otra \\ diferente a la unidad médica nacional que adelanta el CONSIMED, hacia la creación de la \\ Asociación Médica Colombiana-AMC-
}

El desarrollo de la ley 100 de 1993 ha tropezado con un sinnúmero de dificultades como son su gran complejidad, su desconocimiento, aún por las personas que deben aplicarla, la no aplicación en la práctica de sus principios rectores y la incertidumbre de los prestadores del servicio, en particular del talento humano encargado de ejecutarla.

El CONSIMED, entidad que reúne a la Academia Nacional de Medicina, La Asociación Colombiana de Sociedades Cientificas, la Federación Médica Colombiana, ASCOFAME y ASMEDAS, ha elaborado un documento para ser representado a los parlamentarios de las Comisiones VII de Cámara y Senado, con el fin de plantear las cinco grandes dificultades para el desarrollo armónico de la Ley, de tal manera que de no mediar soluciones a dichos problemas, el Sistema General de Seguridad Social en Salud (SGSSS) presenta alto riesgo de colapsarse. Igualmente en el documento se plantean diferentes soluciones posibles.

Para conocimiento de los ginecólogos elaboramos el siguiente resumen ejecutivo, sobretodo teniendo en cuenta que en la Asamblea Nacional de la SCOG celebrada en Medellín los días 27 y 28 de septiembre pasados, se tomó la determinación de participar activamente en el proceso de la. UNIDAD MEDICA NACIONAL promovida por el CONSIMED, a través de la activa y directa participación en el mismo de nuestro Presidente, el Dr. Jaime Ferro Camargo.

Problema 1: Debilidad del sistema de capitación y compensación. El sistema financiero del régimen contributivo se colapsará al participar el ISS en la compensación

En la actualidad el ISS no realiza el ejercicio de la compensación ordenada en el artículo 205 de la Ley 100/ 93, debido a que el actual sistema de información del ISS no permite conocer, con cortes mensuales, las variables básicas requeridas para realizar la capitación y la compensación al sistema, como son: El número exacto de afiliados activos y beneficiarios, su ubicación geográfica y el grupo etáreo al que pertenecen, el monto exacto de la cotización en salud pegados por cada afiliado y el consolidado nacional, los dias y montos pagados por licencias de maternidad e incapacidades.

Para fortuna del Sistema el ISS no compensa. Cuando el ISS participe en el ejercicio de la compensación será la Entidad Promotora de Salud (EPS) deficitaria por excelencia, al tener afiliadas a las personas con ingresos promedios más bajos del mercado.

El ISS exigiría pagos por déficit en la compensación superiores a los ciento veinte mil millones de pesos (para el año 1996) que la subcuenta de compensación no podrá cancelar, si recordamos que los recursos apropiados para el pago a todas las EPS no llegan a los 47 mil millones; en consecuencia el Sistema se colapsará.

Este calculo se realizó con base en una población de 9'319.654 beneficiarios reportados por el ISS a la Supersalud al 31 de diciembre de 1995. Por supuesto, si la realizamos con los 12 millones que el propio ISS afirma tener, la Subcuenta de Compensación le saldria a deber en el presente año más de 200 mil millones de pesos, lo cual reiteramos, colapsará el sistema.

\section{Propuesta 1: Sistema dual en salud}

La Ley 100 creó un sistema dual en pensiones: Por un lado los Fondos Privados de Pensiones (FPP) manejan los aportes bajo los criterios del ahorro individual, lo cual permite a los usuarios acceder a la pensión con el lleno de algunos requisitos especificos establecidos por la Ley. Por el otro lado, el ISS maneja el sistema financiero de la prima media con prestación definida que permite al usuario acceder a la pensión con el lleno de los requisitos de edad y semanas de aportes. Representa el sistema anterior con algunas modificaciones. El Usuario con plena libertad de elección escoge uno de los dos regímenes en competencia: Ahorro individual o prima media.

La propuesta formaliza una situación que parcialmente se está dando de hecho y consiste en crear EL SISTEMA DUAL EN SALUD: Separar el sector público del privado, crear como en pensiones dos regimenes diferentes, en competencia, los cuales serian:

\section{A. Régimen de EPS privada}

Por un lado las EPS privadas manejarán el nuevo sistema financiero creado por la ley 100 para los recursos del Régimen Contributivo, tal como está hoy reglamentado en el Decreto 1283 del 23 de julio de 1996 y cuyas principales características son:

- Cotización del 12\% (2/3 el empleador, 1/3 el trabajador). 
- Girar un punto de la cotización con destino a la subcuenta de Solidaridad del FOSYGA, para financiar el Régimen Subsidiado.

- Destinar 0.5 puntos de la cotización para actividades de Promoción y Prevención, según lo establecido en el artículo 222 de la Ley 100 y reglamentado por el CNSSS (Acuerdo 29).

- Descontar de los ingresos por cotización los gastos pagados por licencias de maternidad e incapacidades, equivalente a 0.3 puntos de la cotización.

- Tomar una doceava parte del valor de la UPC por cada beneficiario.

- Girar a la subcuenta de compensación del FOSYGA los excedentes o cobrar el déficit.

- Con los recursos obtenidos por la UPC, sumados a las cuotas moderadoras, copagos y los rendimientos financieros garantizar la prestación del POS.

- Contratar con IPS, públicas o privadas, propias o ajenas, la prestación de los servicios de salud.

\section{B. Régimen de EPS pública}

Por otro lado, el ISS y las EPS públicas manejarán el sistema de reparto simple, con las siguientes características generales:

- Cotización del 12\% (2/3 el empleador, 1/3 el trabajador).

- La EPS Pública captará la cotización, la cual constituye recursos propios.

- El manejo financiero será con el sistema de reparto simple, el cual en el ISS ha presentado excelentes resultados y nunca, hasta el presente, ha reportado pérdidas.

- La EPS Pública destinará 0.5 puntos de la cotización para financiar los programas de promoción y prevención de la salud, tal como está hoy establecido.

- Con los recursos de cotización la EPS pública garantizará a todos sus beneficiarios medicina familiar integral, tal como se estableció en el Decreto 1650 de 1977.

- La EPS pública no tendrá preexistencias, no cobrará cuotas moderadoras ni copagos y los períodos de carencia serán los existentes en las respectivas normas de cada entidad, anteriores a la Ley 100 de 1993.

- La EPS pública contratará la prestación de los servicios integrales de salud con sus propias IPS, a manera de excepción donde no disponga de la infraestructura necesaria, contratará con IPS externas, públicas o privadas.

Este sistema no es un modelo teórico, ya ha sido aprobado. El ISS antes de la Ley 100 con el 12\% de la

cotización en salud garantizó atención integral acerca del 20\% de su población afiliada: Barranquilla, Medellín, Norte de Santander, entre otros, con excelentes resultados. La separación del ISS del sector privado se está presentando en la práctica. Separarlos formalmente solucionaría el riesgo de colapso del sistema si el ISS resulta deficitario, como lo suponemos.

Problema 2: Ingresos deficientes para el régimen
subsidiado. No se estú cumpliendo el "Paripassu"

Hay un hecho relevante identificado como una de las causas que producen menores ingresos al Régimen Subsidiado y consiste en que actualmente el Gobierno Nacional no está cumpliendo a cabalidad con el mecanismo financiero conocido como "el paripassu" (peso a peso), establecido en el artículo 221 de la Ley 100. Veamos los resultados:

\section{Año de 1995. Ingresos a la subcuenta de solidaridad (Ejecutado)}

$$
\begin{aligned}
& \text { Un punto de cotización del } \\
& \text { Régimen Contributivo } \\
& \text { Aporte Gobierno Nacional }
\end{aligned}
$$

Diferencia

$\$ 198.920^{\prime} 200.000$

$\$ 70.000^{\prime} 000.000$

$\$ 128.920^{\prime} 200.000$

\section{Año de 1996. Ingresos presupuestados}

Un punto cotización del $R$. C. Aporte Gobierno Nacional

Diferencia
$\$ 125.156^{\prime} 231.000$ $\$ 69.678^{\prime} 870.000$

$\$ 55.477^{\prime} 361.000$
Lo cual significa que en estos dos años el Régimen Subsidiado dejará de percibir cerca de 184 mil millones de pesos, los cuales alcanzarían a subsidiar más de dos millones de personas pobres (A un valor de UPC-S de $\$$ 89.625 para 1996).

Para colmo, hemos conocido el proyecto de Ley por el cual se dictan normas tendientes a la racionalización del gasto público, en el cual la tendencia central es la no participación directa de los aportes fiscales de la nación en Fondo de Solidaridad y Garantia (FOSYGA), lo cual disminuiría substancialmente los recursos y demostraria la responsabilidad económica del Estado con el régimen subsidiado.

Respuesta 2: Cómo procurar mayores ingresos a la subcuenta de solidaridad del FOSYGA para financiar el régimen subsidiado

\section{A. Cumplimiento del Paripassu}

No admite discusión el compromiso del Estado en la salud de los pobres. No se debe repetir la historia de 1973 cuando el Estado dejó de aportar al ISS. La propuesta de racionalización del gasto público, por medio de la cual el Gobierno no aporta al régimen subsidiado es inaceptable porque atenta contra las posibilidades futuras de ampliación de cobertura a los hoy denominados vinculados. 


\section{B. El situado fiscal}

De acuerdo con la Ley 60 de 1993, el situado fiscal tendrá como mínimo los siguientes porcentajes: Para 1994: 23\%. Para 1995: 23.5\%. Para 1996: 24.5\%.

Proponemos continuar la curva ascendente en la siguiente forma: Para 1997: 25\%. Para 1998: 26\%. Para 1999: 27\%. Para 2000: 28\%. Para 2001: 30\%.

\section{Problema 3: El futuro incierto de los hospitales}

La ley 100 cambia abruptamente el subsidio a la oferta. por el subsidio a la demanda. Por tres años los hospitales mantendrán el presupuesto histórico global a través de transferencias provenientes del Situado Fiscal, via Minsalud y Servicios Seccionales (Arts. 238 y 239 de la Ley 100).

Estos recursos que hoy representan cerca del $85 \%$, progresivamente se irán recortando a partir de 1997 hasta desaparecer en el año 2001. Las cuotas de recuperación, producto de venta de servicios directos de los hospitales que hoy representan en conjunto cerca de un $15 \%$, progresivamente se irán incrementando hasta que el hospital se financie exclusivamente con recursos por venta de servicios.

El subsidio a la demanda y a la autofinanciación de los hospitales genera estímulos perversos tales como:

Realización de actos médicos, exámenes de laboratorio, procedimientos diagnósticos y terapéuticos no siempre necesarios por la necesidad imperiosa de facturar para subsistir.

Selección adversa de pacientes vinculados y de enfermos con patologías no incluidas en el POS subsidiado.

- Venta y/o liquidación de los Hospitales que no produzcan en el sentido económico.

- Restricción de camas hospitalarias al servicio de la población pobre, para ofrecerlas al régimen contributivo que tiene mejor capacidad de pago.

- Lo más perverso es que la salud no es negocio, el negocio es la enfermedad. Si no hay enfermos no hay a quien facturar. ¿Cuál es el incentivo para generar. programas de promoción y prevención de la salud?

Se desestimula la docencia universitaria por no considerarla costo efectiva.

Estimula una politica de contención de costos a ultranza, lo cual se refleja en medidas tales como:

* Supresión de empleos

* Privatización de servicios e incremento de costos para el usuario

* No prestación de servicios si no existe pago anticipado

* Aumento a ultranza de la productividad, en el sentido equivocado y poco aplicable en salud: Rendi- miento. No es mejor médico aquel quien realiza mayor número de consultas por unidad de tiempo.

Una politica social no puede ser buena, si pasa por el sacrificio de los hospitales que prestan servicios a la población más necesitada, si pasa por el sacrificio de su función social y si pasa por el sacrificio de sus trabajadores.

\section{Propuesta 3: Sistema mixto de subsidio a la oferta y subsidio a la demanda}

Si el sistema tradicional de subsidio a la oferta conlleva un estímulo indirecto a la ineficiencia, el sistema de subsidio a la demanda acarrea estimulos perversos en la práctica médica, como arriba lo anotamos.

Proponemos tomar lo bueno de cada sistema para controlar sus debilidades y constituir un sistema mixto, de tal forma que los subsidios a la oferta se mantengan indefinidamente o por lo menos mientras exista población vinculada, simultáneos con subsidios de demanda, de la siguiente forma.

\section{A. Serán recursos para la oferta:}

Los recursos provenientes del Situado Fiscal, las rentas cedidas a los departamentos y distritos y el $25 \%$ de la participación de los municipios en los ingresos corrientes de la Nación a que hace referencia el numeral 2 del artículo 22 de la Ley 60 de 1993. Se destinarán prioritariamente para garantizar los gastos por servicios personales de todos los servidores públicos y contratistas del sector de la salud.

\section{B. Serán recursos para la demanda:}

Los provenientes del FOSYGA, Subcuenta de Solidaridad, para la afiliación de las familias más pobres, previa afiliación a una Administradora de Régimen Subsidiado (ARS) (Ver gráfica B). Los hospitales con la venta de servicios sufragarán todos los demás costos diferentes a gastos por servicios personales.

\section{Problema 4: Pérdida de derechos adquiridos en salud}

Señala la Ley $100 / 93$ en el art. 162 que para el afiliado cotizante el contenido del POS será el contemplado en el decreto $1650 / 77$ y sus reglamentarios. Es decir, al trabajador se le deberá dar en salud, cuando menos, lo que ofrecía el ISS antes del 23 de diciembre de 1993. Esta clara directriz juridica no es respetada en la reglamentación del POS, como trataremos de demostrarlo a continuación:

\section{Período de protección laboral}

Con base en la norma anterior el ISS al asegurado que haya sido desafiliado le cubre las prestaciones asistenciales en forma integral, lo mismo que al trabajador activo, hasta por el término de dos meses (en la práctica son tres meses), contados a partir de la fecha de su desafiliación (art. 11 del decreto 770/75): Este derecho quedó limitado a un mes, siempre y cuando el 
trabajador haya estado afiliado como mínimo 6 meses a la respectiva EPS y sólo serán atendidas aquellas patologías que venían en curso de tratamiento (art. 25 del decreto 1938/94).

\section{Protección especial por maternidad}

Así mismo, el ISS da protección especial por maternidad consistente en la atención prenatal, parto y puerperio, lo mismo que la atención del recién nacido (R.N.) durante el primer año de vida, dado que la Ley señala que el R.N. debe quedar automáticamente inscrito en la EPS donde la madre fue atendida, si la concepción tuvo lugar antes de la fecha del retiro y cuando la asegurada (cotizante, esposa o compañera permanente) hubiere aportado un mínimo de cuatro semanas. (Art. 23, 24 y 26 del Decreto 770/75). Esta prestación quedó limitada a un mes, es decir, si la trabajadora (o la esposa o compañera permanente del trabajador) no tiene el parto un mes después de perder el empleo, pierde el derecho a la atención del parto y de paso el R.N. también es excluido.

\section{Los pensionados}

Anteriormente el ISS y las diferentes Cajas de Previsión ofrecian atención integral a los pensionados, sin exclusiones o limitaciones, sin cobro de cuotas moderadoras, ni planes complementarios a cargo del pensionado, los cuales se introducen con la Ley 100/93 y sus decretos reglamentarios. Someterlos a exclusiones que anteriormente no tenían, exigirles cuotas moderadoras que anteriormente no pagaban o supeditarlos a limitaciones tecnológicas u hoteleras para obligarlos a adquirir costosos Planes de Atención Complementaria en Salud, con los escasos recursos de los pensionados; es un atentado contra los derechos en salud adquiridos antes de entrar en vigencia la Ley 100/93.

\section{Propuesta 4: Respeto integral a los derechos adquiridos en salud}

Se debe dar cumplimiento al artículo 162 de la Ley 100, el cual señala: "Para los afiliados cotizantes el contenido del POS que defina el CNSSS será el contemplado en el Decreto Ley 1650 de 1977 y sus reglamentaciones, incluyendo la provisión de medicamentos en su presentación genérica..."

El estudio realizado por el Dr. Wolff publicado en agosto del presente año, compara el contenido del manual de tarifas del ISS, con el contenido del POS establecido en el Decreto 1938, encontrando que el número de actividades, intervenciones y procedimientos prestados por el ISS en las especialidades médico quirúrgicas son 1892, en tanto que el POS contiene solamente 1.276, con una diferencia a favor del ISS de 602.

En procedimientos diagnósticos y terapéuticos el ISS realiza 1.779, en tanto el POS sólo incluye 1.014. Diferencia 765 . En servicios ambulatorios de salud oral el ISS realiza 80 y el POS solamente 26. Diferencia 54. En el listado de medicamentos del ISS existen 102 productos farmacéuticos que no figuran en el POS. En el Manual de Medicamentos del POS existen medicamentos que no incluye el ISS por considerarlos no pertinentes desde el punto de vista técnico y científico. Hay 33 productos del manual del POS que no tienen proveedor en el pais.

\section{Problema 5: Bajo nivel salarial del sector y falta de estímulos a los profesionales de la salud}

Los actores principales del proceso de la Seguridad Social en el régimen contributivo son LOS USUARIOS, LAS EPS, LAS IPS Y LOS PROFESIONALES Y TRABAJADORES DE LA SALUD, bajo la dirección, control y coordinación del Gobierno y con la financiación de los aportes PARAFISCALES obrero patronales. Es necesario un perfecto equilibrio entre los diferentes actores en su capacidad de expresión y negociación, de lo cual el Estado debe ser un garante imparcial.

Las tarifas más representativas y reguladoras del mercado, las del ISS y las del SOAT, son muy bajas y no compensan la responsabilidad y la complejidad del acto médico. Estas tarifas son impuestas unilateralmente, sin concertación con los representantes de los prestadores del servicio, lo cual hipertrofia el poder de las EPS en detrimento de los demás actores.

En los hospitales del sector público, comprometidos esencialmente con el Régimen Subsidiado la situación laboral es más critica: Desafortunadamente el Decreto 439 de 1995 no solucionó la inequidad existente en la remuneración de los trabajadores del sector de la salud, con relación a otras áreas del sector público.

Los contratos de prestación de servicios personales utilizados por las Empresas Industriales y Comerciales del Estado, en particular el ISS, son una forma aberrante de disfrazar una relación laboral formal y evitar el pago de prestaciones laborales de Ley.

\section{Propuesta 5: Concertación}

Las manifestaciones de confrontación de los profesionales de la salud, en particular los médicos, con las EPS conllevan traumas innecesarios en el desarrollo del Sistema y en la prestación de los servicios de salud. Esto se puede evitar, en primer término, favoreciendo los procesos de concertación entre las diferentes EPS, IPS, Cuerpo Médico y usuarios, con la coordinación del Ministerio de Salud.

La concertación es una de las reglas del servicio público de salud, rectoras del SGSSS, establecida en el artículo 153 de la Ley. Por esta razón el CONSIMED está dispuesto a colaborar en el proceso de desarrollo del sistema, siempre y cuando sea tenido en cuenta el punto de vista de los médicos.

Como la inequidad salarial en el sector de la salud continúa latente, para solucionarla se requiere ordenar por vía de la Ley una auténtica nivelación, actualización e incremento salarial, como ha sido reconocido por 
el propio Congreso de la República y lo han planteado el CONSIMED y las organizaciones sindicales de los trabajadores de la salud.

Merece especial mención y respaldo del CONSIMED el pronunciamiento hecho por el Concejo General de Educación Médica de ASCOFAME, en relación con las politicas estatales atinentes a la formación del recurso humano para el área de la salud, en particular en el campo médico, las cuales aunque no hacen parte de la reglamentación de la Ley 100, si tienen mucho que ver con el desarrollo del sistema.

Una de esas politicas, coincidente con la autonomía universitaria prescrita en la Constitución Politica de 1991 y en la Ley 30 de 1992, ha sido la de permitir la apertura indiscriminada de programas de formación médica ante la perspectiva de que tal recurso humano vaya a necesitarse en gran escala para atender la masiva demanda de servicios que se avecina. Esta política apunta a darle solución a un asunto cuantitativo de mercado para favorecer la oferta, haciendo abstracción de lo cualitativo. Tal desacuerdo es lo que en verdad preocupa a ASCOFAME y al CONSIMED.

Por lo anteriormente expuesto el CONSIMED se suma a la solicitud de ASCOFAME en el sentido de que se haga un replanteamiento sobre las politicas de apertura de nuevos programas de Medicina, como también de la vigilancia y control de los ya autorizados.

\section{HERMAN REDONDO GÓMEZ}

Asesor de la SCOG en asuntos gremiales

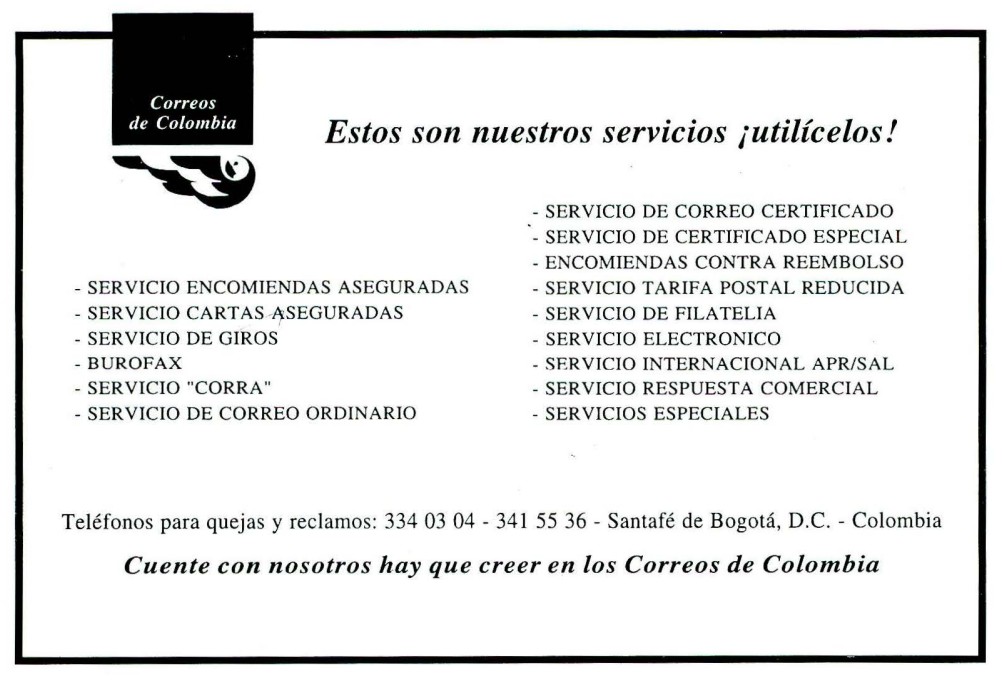

